\title{
Protein microspheres as suitable devices for piroxicam release
}

\author{
Raquel Silva ${ }^{a}$, Helena Ferreira ${ }^{\mathrm{a}, \mathrm{c}}$, Ana C. Carvalho ${ }^{\mathrm{b}}$, Andreia C. Gomes ${ }^{\mathrm{b}}$, Artur Cavaco-Paulo ${ }^{\mathrm{a}, *}$ \\ a University of Minho, Department of Textile Engineering, Campus de Azurém, 4800-058 Guimarães, Portugal \\ b CBMA (Centre of Molecular and Environmental Biology), Department of Biology, University of Minho, Campus of Gualtar, 4710-057 Braga, Portugal

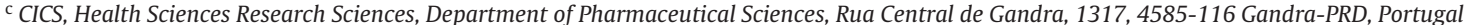

\section{A R T I C L E I N F O}

Article history:

Received 14 July 2011

Received in revised form 28 October 2011

Accepted 30 November 2011

Available online 8 December 2011

\section{Keywords}

Drug delivery systems

Controlled release

Piroxicam

Protein microspheres

Sonochemistry

\begin{abstract}
A B S T R A C T
Bovine serum albumin-piroxicam (BSA-piroxicam) and human serum albumin-piroxicam (HSApiroxicam) microspheres were sonochemically prepared and characterized. The use of polyvinyl alcohol (PVA) lead to an improvement of formulation characteristics, including smaller size, lower polydispersity index (PDI), higher entrapment efficiency and higher stability. The release kinetics of these proteinaceous microspheres was determined in presence of protease, indicating an anomalous drug transport mechanism (diffusion and polymer degradation). In presence of higher protease concentration, BSA microspheres exhibit Case II transport, leading to zero order release (protein degradation). These proteinaceous devices did not show cytotoxicity against human skin fibroblasts in vitro, for range concentrations below to $300 \mathrm{mg} \mathrm{L}^{-1}$, greatly supporting their potential application in the treatment of inflammatory diseases.
\end{abstract}

(C) 2011 Elsevier B.V. All rights reserved.

\section{Introduction}

Non-steroidal anti-inflammatory drugs (NSAIDs) are the most widely used drugs in the treatment of inflammatory diseases, such as rheumatic disease, but are also being increasingly used for nonrheumatic conditions, including acute and chronic pain, biliary and ureteric colic, dysmenorrheal inflammation and fever [1]. The mechanism of action of NSAIDs is mediated by its capacity to inhibit cyclo-oxygenase (COX) activity. The COX enzyme can be divided into two isoforms - a constitutive isoform (COX-1), which is responsible for maintaining normal function in the gastrointestinal and renal tracts, for example, and an inducible isoform (COX-2), which is found in areas of inflammation and in the brain [2]. It has been suggested that the anti-inflammatory actions of NSAIDs are due to the inhibition of COX-2, whereas the unwanted side-effects are due to the inhibition of COX-1 [3-5]. The vast majority of NSAIDs currently available is not selective for COX-2 and can thus cause the adverse reactions so commonly seen during NSAIDs treatment [3-5]. Therefore, the entrapment in microspheres of the NSAIDs, such as piroxicam, which are not selectively targeting COX-2, could be useful to minimize their adverse reactions. Various types of macromolecular substances, such as synthetic and natural polymers have been used in drug delivery research, as they can effectively deliver the drug to target the action site and thus increasing the thera-

\footnotetext{
* Corresponding author. Tel.: +351253 51027; fax: +351 253510293.

E-mail address: artur@det.uminho.pt (A. Cavaco-Paulo).
}

peutic benefit, while minimizing the side effects [6-10]. Recently, protein microspheres have been shown efficacy as biodegradable and biocompatible carrier, which can incorporate a variety of drugs in relatively non-specific fashion [11-15]. Albumin is a promising material and has been extensively investigated as drug delivery system, because of its biodegradability and low toxicity $[16,17]$. In addition, albumin proteins has a functionality for transporting different macromolecules in the bloodstream to target organs making it a potential macromolecular carrier for the site-directed delivery of drugs [18]. Four of the most important characteristics of particles are their size (presence of low polydispersity, bellow 0.1 ), entrapment efficiency, zeta-potential (surface charge), and the release characteristics [19]. Previous studies demonstrate that 3 min of sonication promote the entrapment of different components, such as antibiotic (tetracycline) [20], anti-cancer drugs (taxol and gemcitabine) $[11,12]$ or even RNA molecules [21] in bovine serum albumin (BSA) microspheres with approximately $2 \mu \mathrm{m}$ of diameter with a high polydispersity index. However, the profile as well as the mechanism for the release of bioactive components at the targeted environment is not clarified. The main aim of this research was to study the efficiency of the sonochemical method for the entrapment of piroxicam in protein microspheres, using bovine or human serum albumin (HSA) as starting material, and to study the possibility of using the proteinaceous devices as a drug-delivery agent, determining their release kinetics in the presence of protease. Moreover, further miniaturization of the microspheres, to improve their colloidal stability and the entrapment efficiency of drug, was also performed via different stabilizers: polyvinyl alcohol (PVA), pluronic acid F68 (pluronic F68) and tween 80. Herein, 
this approach was applied in order to obtain particles with small diameters and a monodisperse population (polydispersity index lower than 0.1 ) increasing their surface area promoting at the same time a faster drug released. The ability of proteins to form microspheres was evaluated by quantifying protein concentration with the Lowry assay on the supernatant after ultrasound treatment [22]. Particle size, as well as the polydispersity index (PDl), of microspheres, was evaluated by photon correlation spectroscopy (PCS). It is well known that the particle size can significantly affect the microspheres properties and is important for their interaction with the biological environment [23]. Particle size values are thus crucial in the development and optimization of preparation process. In addition the zeta-potential of microspheres was also evaluated by electrophoretic laser Doppler anemometry, to obtain an indication of surface potential. The scanning transmission electron microscopy (STEM) has been employed to characterize the overall structure and shape of the microspheres. The entrapment efficiency of piroxicam in microspheres was assessed and the release profile was evaluated in the presence of a protease. Finally, microspheres were screened for cytotoxicity using a metabolic assay on an human fibroblast cell line.

\section{Materials and methods}

\subsection{Materials}

The anti-inflammatory drug, piroxicam, was acquired from Sigma (Spain), as well as the PVA (Mw $=30.000-70.000 \mathrm{Da}$, and $87-90 \%$ hydrolyzed), tween 80 and pluronic F68. The BJ5ta cell line (telomerase-immortalized human normal skin fibroblasts) was purchased from the European Collection of Cell Cultures (ECACC) and cultured according to ATCC recommendations. The centricon tubes (molecular-weight cut-off of $100 \mathrm{kDa}$, Amicon) were purchased from Amicon. All other reagents, including those used in the cell culture, were analytical grade and purchased from Sigma (Spain).

\subsection{Equipment}

The experimental set up used was composed of a probe type ultrasound source (20 kHz Sonics \& Materials Vibracell CV 33) fitted with a $3 \mathrm{~mm}$ diameter titanium micro-tip. Power delivery was controlled as percentage amplitude. The reaction vessel was an open glass cell (diameter $19 \mathrm{~mm}$ and height $75 \mathrm{~mm}$ ), which contained $16 \mathrm{~mL}$ of sample solution. The sonochemical reactor temperature was controlled via a thermo-stated water bath with a freezer exchanger placed within a thermo jacket cell; this gave a steady operating temperature of $10 \pm 1{ }^{\circ} \mathrm{C}$. Temperature was monitored throughout using K type thermocouples (TC).

\subsection{Sonochemical preparation of proteinaceous microspheres}

Microspheres, with or without anti-inflammatory drug, were synthesized by an adaptation of the Suslick and Grinstaff method [24]. The piroxicam (3 mM) was added to protein solution $\left(5 \mathrm{~g} \mathrm{~L}^{-1}\right)$ and layered with vegetable oil. The very low solubility of piroxicam required the use of dimethyl sulfoxide:water solutions $(1: 99 \mathrm{v} / \mathrm{v})$. When surfactants were used as stabilizer, they were dissolved in aqueous phase. The bottom of the high-intensity ultrasonic horn was positioned at the aqueous/organic interface employing an amplitude of $40 \%$ with a temperature of $10 \pm 1{ }^{\circ} \mathrm{C}$ and with a total treatment of $3 \mathrm{~min}$. The separation of phases was accomplished in a few minutes, due to the lower density of microspheres, relative to the density of water. After, microspheres were collected by centrifugation $(2000 \times g, 30 \mathrm{~min})$ using the centricon tubes. Finally, the microspheres were sterilized with UV radiation over $2 \mathrm{~h}$.

\subsection{Determination of microspheres yield}

The efficiency of microspheres formation was performed via Lowry method [22], using BSA as standard. This method was based on the determination of protein in the supernatant. The efficiency of microspheres formation was calculated as follows:

Microspheres formation $(\%)=\frac{[\text { Protein }]_{\text {total }}-[\text { Protein }]_{\text {supernatant }}}{[\text { Protein }]_{\text {total }}} \times 100$

where [Protein $]_{\text {total }}$ and [Protein $]_{\text {supernatant }}$ is the initial and the final protein concentration in the aqueous solution, respectively.

\subsection{Determination of size and zeta-potential}

The zeta-potential and the size distribution of microspheres were determined at $25.0 \pm 0.1{ }^{\circ} \mathrm{C}$ using a Malvern zetasizer NS (Malvern Instruments) by electrophoretic laser Doppler anemometry and photon correlation spectroscopy (PCS), respectively. The proteinaceous microspheres were diluted $1: 100$, and in order to verify the reproducibility of the proteinaceous microspheres preparation assembled under the typical process described above, the procedure was carried out at least three times for each of the individual microspheres. The results were expressed as mean value \pm standard deviation.

\subsection{Scanning transmission electron microscopy (STEM)}

For STEM analysis, the diluted microspheres suspension was dropped in Copper grids with carbon film 400 meshes, $3 \mathrm{~mm}$ diameter. The shape and morphology of microspheres were observed using a NOVA Nano SEM 200 FEI.

\subsection{Entrapment efficiency of piroxicam}

Entrapment efficiency of piroxicam in proteinaceous microspheres was measured by UV spectrophotometry, at $353 \mathrm{~nm}$ using a He $\lambda$ ios $\gamma$ ThermoSpectronic spectrophotometer (Unicam). The separation of free piroxicam was achieved by centrifugation of samples at $2000 \times \mathrm{g}$ for $30 \mathrm{~min}$, using the centricon tubes (molecular-weight cut-off of $3 \mathrm{kDa}$ ). The entrapment efficiency of piroxicam was determined as follow:

Entrapment $(\%)=\frac{[\text { Piroxicam }]_{\text {initial }}-[\text { Piroxicam }]_{\text {final }}}{[\text { Piroxicam }]_{\text {final }}} \times 100$

where [Piroxicam $]_{\text {initial }}$ is the initial concentration of piroxicam used and $[\text { Piroxicam }]_{\text {final }}$ is the final concentration of piroxicam in supernatant. Measurements were recorded in triplicate and the results were expressed as mean value \pm standard deviation.

\subsection{In vitro piroxicam release profile from proteinaceous microspheres}

The enzymatic degradation of the developed carrier systems was investigated by incubating the microspheres in phosphate buffered saline solution (PBS, $0.01 \mathrm{M}, \mathrm{pH}=7.4$ ) with different concentrations of protease, subtilisin from Bacillus sp. (E.C. 3.4.21.62), $\left(0.075,0.50\right.$ and $\left.3.0 \mathrm{U} \mathrm{mL}^{-1}\right)$. The selected enzyme was used to mimicking the enzymatic environment of the human body and as a trigger for drug release. The microspheres were incubated at room temperature under constant shacking (50-60 rpm). At determined time points, aliquots were taken and the piroxicam release was monitored by absorbance measurements at wavelength of $353 \mathrm{~nm}$ using a He $\lambda$ ios $\gamma$ ThermoSpectronic spectrophotometer (Unicam). 
The quantification of the release was established by a standard absorbance curve. All the release experiments were carried out in triplicate and for a period of 6 days. Results are reported as average \pm standard deviation.

\subsection{Drug release kinetics}

The release behavior of compounds from polymeric systems can be determined by fitting the release data to the empirical relationship given by the Ritger-Peppas equation:

$\frac{M_{t}}{M_{\infty}}=k t^{n}$

where $M_{t} / M_{\infty}$ is the fractional drug release at time $t$; $t$ is the release time; $k$ is the kinetic constant, and $n$ is the diffusion exponent characteristic of the release mechanism. To determine $n$ values for proteinaceous microspheres, Eq. (3) is transformed in Eq. (4), and $n$ is determined from the slope of the plot of log (\% release) versus $\log t$.

$\log (\%$ released $)=\log \left(\frac{M_{t}}{M_{\infty}}\right)=\log k+n \log t$

\subsection{Cytotoxicity evaluation}

The cytotoxicity of BSA and HSA microspheres, containing piroxicam, was evaluated using murine embryonic fibroblasts cells (MEFs), isolated from E13.5 embryos and normal human skin fibroblasts (BJ5ta), from American type culture collection (ATCC). The BJ5ta cell line (normal human skin fibroblasts) was maintained according to ATCC recommendations (4 parts Dulbecco's modified Eagle's medium (DMEM) containing $4 \mathrm{mM}$ L-glutamine, $4.5 \mathrm{~g} \mathrm{~L}^{-1}$ glucose, $1.5 \mathrm{~g} \mathrm{~L}^{-1}$ sodium bicarbonate and 1 part of Medium 199, supplemented with $10 \%(\mathrm{v} / \mathrm{v}$ ) of fetal bovine serum (FBS), $1 \%(\mathrm{v} / \mathrm{v})$ of Penicillin/Streptomycin solution and $10 \mu \mathrm{g} \mathrm{mL}^{-1}$ hygromycin B). The cells were maintained at $37^{\circ} \mathrm{C}$ in a humidified atmosphere of $5 \% \mathrm{CO}_{2}$. Culture medium was refreshed every 2-3 days. Cells were seeded at a density of $10 \times 10^{3}$ cells $/ 100 \mu \mathrm{L} /$ well on 96 -well tissue culture polystyrene (TCPS) plates (TPP, Switzerland), in the day before of experiments, and then exposed to different microspheres concentrations, as well as to different controls solutions (BSA and HSA solutions, vegetable oil; PVA solution; piroxicam solution) added to fresh culture medium. At 24,48 and $72 \mathrm{~h}$ of exposure, cell viability was determined using the alamarBlue ${ }^{\circledR}$ assay (Invitrogen, EUA). $10 \mu \mathrm{L}$ of alamarBlue compound was added to each well containing $100 \mu \mathrm{L}$ of culture medium. After $4 \mathrm{~h}$ of incubation at $37^{\circ} \mathrm{C}$ the absorbance at $570 \mathrm{~nm}$, using $600 \mathrm{~nm}$ as a reference wavelength, was measured in a microplate reader (Spectramax 340PC). The quantity of resorufin formed is directly proportional to the number of viable cells. Data are expressed as means with standard errors of the means. Two-way ANOVA followed by post hoc Bonferroni test (GraphPad Prism 5.0 for Windows) was employed with statistically significant differences when $P<0.05$. The samples were tested at least in triplicates per two independent experiments.

\section{Results}

\subsection{Influence of stabilizer addition in microspheres characterization parameters}

In this work, matrices based on BSA or HSA proteins, were produced and characterized. To ensure microspheres suitability for the intended type of application and to enable a focused development of dispersions with specific properties, the characteristics of the dispersions have to be known in detail.
Table 1

Effect of PVA on Z-average (nm), PDI and zeta-potential (mV) values of BSA microspheres.

\begin{tabular}{cccc}
\hline PVA amount (\%) & Z-Average $(\mathrm{nm})$ & PDl & Zeta-potential $(\mathrm{mV})$ \\
\hline 0 & $402.9 \pm 65.1$ & $0.44 \pm 0.03$ & $-15.6 \pm 1.5$ \\
2 & $392.7 \pm 20.1$ & $0.35 \pm 0.01$ & $-4.7 \pm 0.3$ \\
4 & $378.1 \pm 40.6$ & $0.18 \pm 0.08$ & $-3.9 \pm 0.6$ \\
6 & $340.4 \pm 15.6$ & $0.15 \pm 0.06$ & $-3.8 \pm 0.2$ \\
8 & $257.9 \pm 5.90$ & $0.06 \pm 0.04$ & $-3.9 \pm 0.1$ \\
10 & $259.1 \pm 4.20$ & $0.06 \pm 0.06$ & $-3.7 \pm 0.3$ \\
\hline
\end{tabular}

Values reported are the mean \pm SD of at least three different batches of each microsphere formulation.

In a previous study [25] it was demonstrated that smaller sizes are obtained with 95/5 (\%) of aqueous/organic phase. To overcome the drawback of polydisperse population, the addition of different stabilizers, on formulations was evaluated. One key parameter to obtain small particles with a low PDl is the type of surfactant/stabilizer used. A wide range of synthetic and natural molecules with varying properties has been proposed to prepare particles. Nevertheless, for parenteral and topical application nonionic surfactants, such as tween 80, pluronic F68 and PVA, are generally used [26]. However, PVA have a surfactant character but its stabilizing effect is exerted not through boundary surface adsorption but by increasing the viscosity of water [27-29].

Firstly, the efficiency of microspheres formation was monitored with the Lowry assay [22]. The addition of stabilizer for the production of protein devices did not affect the yield of microspheres formation $(\approx 100 \%)$. The effect of stabilizers on microspheres size was then evaluated. In this experiment, PVA was the first adopted stabilizer and BSA microspheres of different sizes were successfully prepared controlling PVA amount (Table 1).

Results show that when PVA quantity is increased, the size of proteinaceous microspheres becomes much smaller. From Table 1, it is possible to verify that not only the size of BSA microspheres gets effectively smaller, within a certain range, with the increasing of PVA amount, but also the particle size distribution, determined as PDl, becomes narrower. There are two main explanations for these observations. Firstly, the tiny particles make wavy movement and collide each other in an emulsion leading to particles aggregation. The polymerization degree of PVA used in the experiment can change the viscosity of an aqueous solution decreasing the wavy movement speed and the collision of the tiny particles. Secondly, the adsorption of PVA on the surface of proteinaceous microspheres can lower the surface energy and further agglomeration of proteinaceous microspheres $[28,30]$. The optimization of stabilizer amount was achieved at $8 \%$, as higher percentages of PVA did not present significant change in microspheres size and PDI.

Besides PVA, others macromolecular stabilizers have been tested, namely, pluronic F68 and tween 80. The same behavior was observed when different amounts of pluronic F68 and tween 80 were added to the formulation (data not shown). In Table 2 it is compared the effect of the three stabilizers used on the $Z$-average, PDl and zeta-potential values of the particles. PVA presented an high ability to reduce the particle sizes and, at the same

Table 2

Effect of different stabilizers, PVA (8\%), pluronic F68 (8\%) and tween $80(8 \%)$, on $\mathrm{Z}$-average $(\mathrm{nm})$, PDI and zeta-potential $(\mathrm{mV})$ values of BSA microspheres.

\begin{tabular}{lcrc}
\hline Stabilizers $(8 \%)$ & Z-Average $(\mathrm{nm})$ & \multicolumn{1}{c}{ PDl } & Zeta-potential $(\mathrm{mV})$ \\
\hline PVA & $247.9 \pm 5.9$ & $0.060 \pm 0.04$ & $-3.9 \pm 0.1$ \\
Pluronic F68 & $392.1 \pm 60.1$ & $0.29 \pm 0.05$ & $-2.9 \pm 0.4$ \\
Tween 80 & $386.3 \pm 17.1$ & $0.28 \pm 0.06$ & $-3.5 \pm 0.1$ \\
\hline
\end{tabular}

Values reported are the mean \pm SD of at least three different batches of each microsphere formulation. 
time, decrease the PDl, promoting a monomodal distribution of BSA microspheres. Therefore, it was used as standard $8 \%$ of PVA for all subsequent preparations. Apart from size determination, zeta-potential was also evaluated to obtain an indication of microspheres surface potential. The surface potential of the particles cannot be measured directly, the zeta-potential (electrical potential at the surface of hydrodynamic shear around the colloidal particle) is usually determined as a characteristic parameter for the particle charge [31,32]. The BSA microspheres possess a zeta-potential around $-15 \mathrm{mV}$, suggesting the presence of negative charge on the microspheres surfaces (Table 2). Nevertheless, the zeta-potential of the particles decreased significantly in the presence of stabilizers (Tables 1 and 2).

A survey of the literature reveals that the measurement of zetapotential allows predict about the stability for colloidal dispersion [33-35]. In general, particle aggregation of charged particles (high zeta-potential) is less likely to occur, due to electric repulsion. However, this rule cannot be strictly applied to the systems containing steric stabilizers, because the adsorption of the steric stabilizers will decrease the zeta-potential, due to the shift in the shear plane of the particle [36].

HSA was also tested in this work and the results obtained substantiate that HSA contributed to a small increase on the $Z$-average value ( $\approx 300 \mathrm{~nm}$ of $Z$-average and 0.070 of $P D l)$, when compared with BSA. In spite of the $80 \%$ of homology, present on the primary structures of the two proteins, the replacement of some hydrophobic amino acids by others (e.g. HSA has a single Tryptophan amino acid while BSA holds two Tryptophan's in its sequence [37]), can explain this difference on the size obtained, once that, the properties of each amino acid demonstrated to be essential in the physico-chemical parameters of microspheres [25,38]. Conversely, the zeta-potential of microspheres prepared with $8 \%$ of PVA and BSA or HSA is very similar $(\approx-4 \mathrm{mV})$.

\subsection{Piroxicam entrapment}

The entrapment efficiency of piroxicam into BSA or HSA microspheres was assessed spectrophotometrically. Entrapment efficiency refers to the ratio of quantity entrapped/adsorbed drug in relation to the total (theoretical) amount of drug used for microspheres production. Fig. 1 shows that PVA proved to be successful in enhancing drug incorporation efficiency in both types of proteinaceous devices prepared. This can be attributed to the fact that

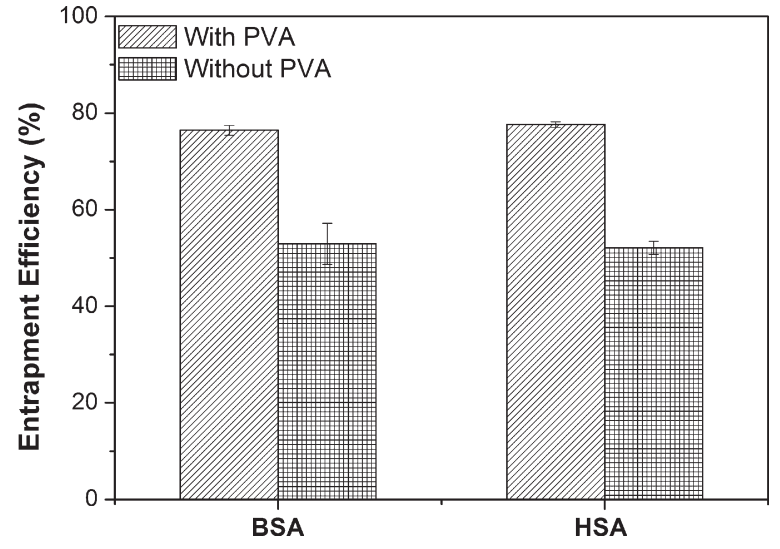

Fig. 1. Effect of PVA (8\%) on the entrapment efficiency of piroxicam (initial concentration of $3 \mathrm{mM})$ into BSA $\left(5 \mathrm{~g} \mathrm{~L}^{-1}\right)$ or HSA $\left(5 \mathrm{~g} \mathrm{~L}^{-1}\right)$ microspheres. Values express the mean results \pm SD values of three different batches.

the presence of PVA decreases the size of particles leading to an enhancing of the surface area, and as a consequence more piroxicam molecules are entrapped into proteinaceous microspheres.

Piroxicam entrapment efficiency is an important factor, once that the release rate is usually dependent on drug concentration gradient [39]. Furthermore, higher levels of drug incorporation lead to a wider concentration gap between the polymeric/protein microspheres and the release medium and can cause a higher diffusion rate. As previously demonstrated, the PVA addition led to a submicron size and a relatively narrow particle size distribution. The drug incorporation in microspheres resulted in a slightly bigger particles with similar PDl values (Fig. 2). The zeta-potential results (Fig. 3) evidence that the presence of piroxicam did not affect significantly the surface charge of proteinaceous microspheres.

\subsection{Stability studies}

Since those colloidal particles can suffer destabilization over time [40], particularly when stored in an aqueous dispersion, particle size was monitored for two months after preparation in order to evaluate their physical stability. The zeta-potential was also determined, since this parameter can change over time, due to degradation of components of the colloidal particles [41]. In an electrostatically stabilized dispersion, charges generated on

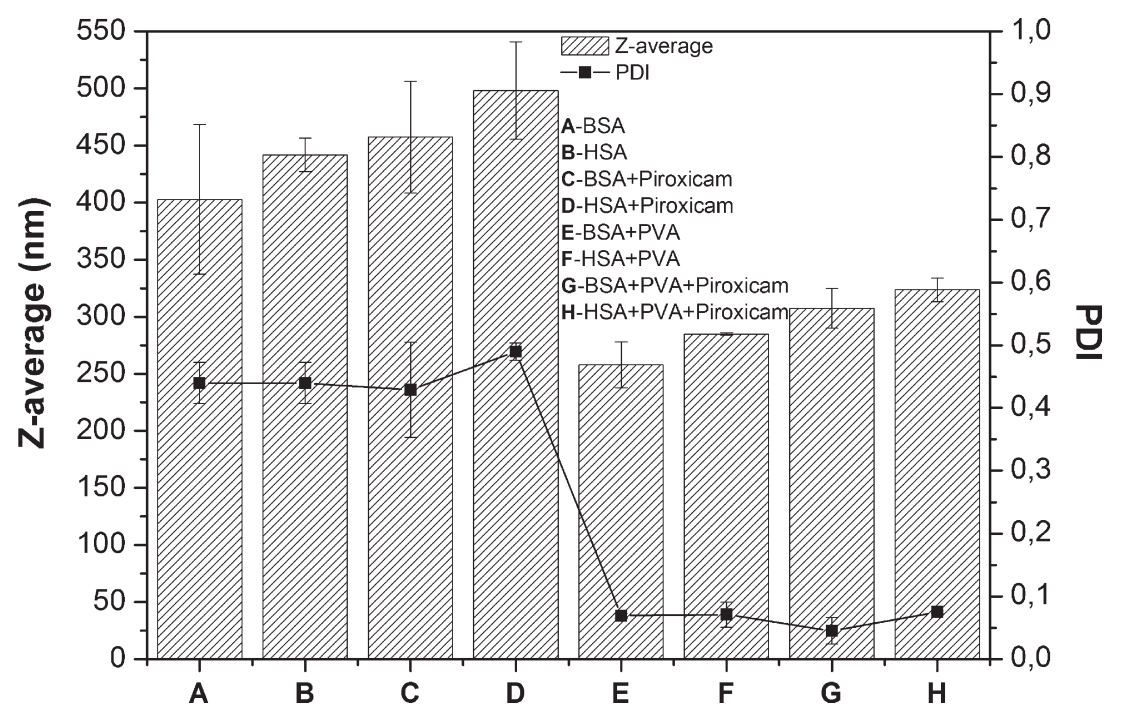

Fig. 2. Z-Average ( $\mathrm{nm}$ ) and PDl of different formulations, prepared with BSA ( $\left.5 \mathrm{~g} \mathrm{~L}^{-1}\right)$ or HSA ( $\left.5 \mathrm{~g} \mathrm{~L}^{-1}\right)$, piroxicam ( $\left.3 \mathrm{mM}\right)$, with or without PVA ( $8 \%$ ). Values reported are the mean $\pm S D$ of at least three different batches of each microsphere formulation. 


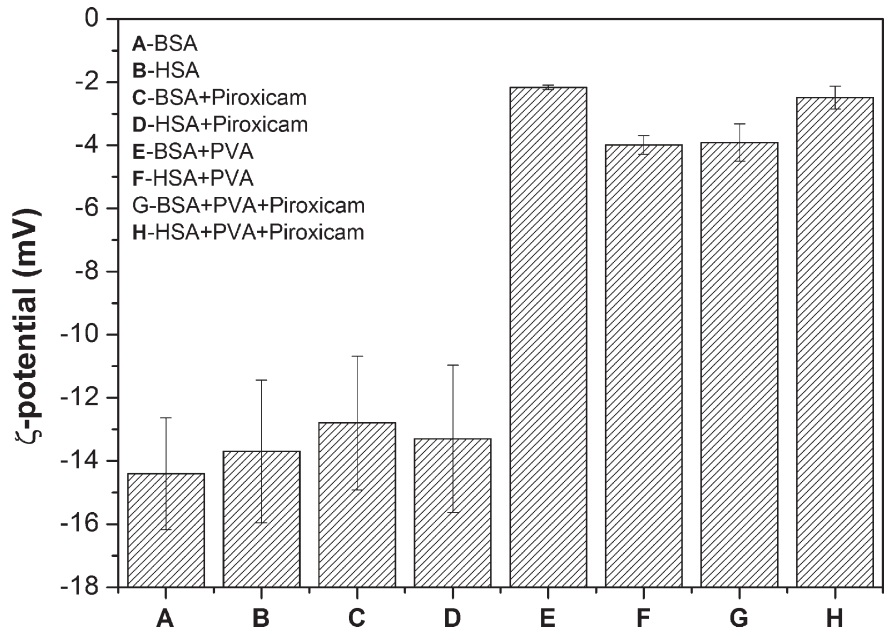

Fig. 3. Zeta $(\zeta)$-potential $(\mathrm{mV})$ of different formulations prepared with BSA $\left(5 \mathrm{~g} \mathrm{~L}^{-1}\right)$ or HSA $\left(5 \mathrm{~g} \mathrm{~L}^{-1}\right)$, piroxicam $(3 \mathrm{mM})$, with or without PVA $(8 \%)$. Values reported are the mean \pm SD of at least three different batches of each microsphere formulation.

the surface of particles prevent or control agglomeration. Steric stabilization takes place when large molecules adsorb onto the surface of particles, thus introducing physical barriers between them [33]. A combination of electrostatic and steric mechanisms produces electrosteric stabilization preventing microspheres agglomeration.

Fig. 4 demonstrates that the presence of PVA increases microspheres' stability, as the size and zeta-potential measurements were roughly stable over time. Conversely, the entrapment of piroxicam without PVA leads to an increase on $Z$-average values and decrease zeta-potential values $(\approx 0 \mathrm{mV})$, after one week of storage. Furthermore, the stabilization of microemulsion with PVA is attributed to the adsorbed chain molecules of PVA on the surface, which have ceaseless thermal motion, resulting in dynamically rough surface preventing coalescence by repulsive entropic force.

\subsection{Morphology}

STEM photographs, in Fig. 5, illustrate that the obtained BSA microspheres are clearly spherical homogeneous particles with smooth surfaces. It was possible to verify that the use of stabilizers (PVA) promoted some restrictions on visualization of microspheres, due to their surface tension. The morphology of HSA microspheres was also evaluated and presents a similarly spherical shape. This morphology would offer the highest potential for controlled release and protection of incorporated drugs, as they provide minimum contact with the aqueous environment, as well as the longest diffusion pathways. Comparing particles with any other shape, spherical particles also require the smallest amount of surface-active agent for stabilization, because of their small specific surface area [23]. These results are also in agreement with PCS results, once that the particle size distribution was very similar.

\subsection{In vitro release profile}

The two developed systems in this study aimed to be used as carriers of an anti-inflammatory molecule are: BSA-piroxicam microspheres and HSA-piroxicam microspheres. One important and desired property is biodegradation when designing drug delivery systems. Biodegradable polymers are able to release the entrapped drug as their degradation take places. Thus, by controlling the degradation rate of polymeric materials the drug release can be identically monitored. For particulate drug delivery systems the enzymatic degradation is a crucial parameter for the release of an embedded drug. However, it should be noted that in vitro release profile obtained might not be quantitatively predictive of the in vivo behavior. Nevertheless, in vitro release studies allowed for screening and rank-ordering various formulations by the rates of drug release and provide a basis for in vivo studies [42]. The release profile of BSA or HSA-based particles, prepared with $5 \mathrm{~g} \mathrm{~L}^{-1}$ of protein, $8 \%$ of PVA and with $3 \mathrm{mM}$ of piroxicam, in the presence of protease enzyme are presented in Fig. 6 . The release can be divided into two phases: an initial burst in the first hours and a continuous release over the remaining time. The burst release of piroxicam is associated with those piroxicam molecules dispersing close to microspheres surface, which diffuse out in the initial incubation time. According with the literature piroxicam revealed a higher partition capacity in lipid bilayer and consequently present an higher

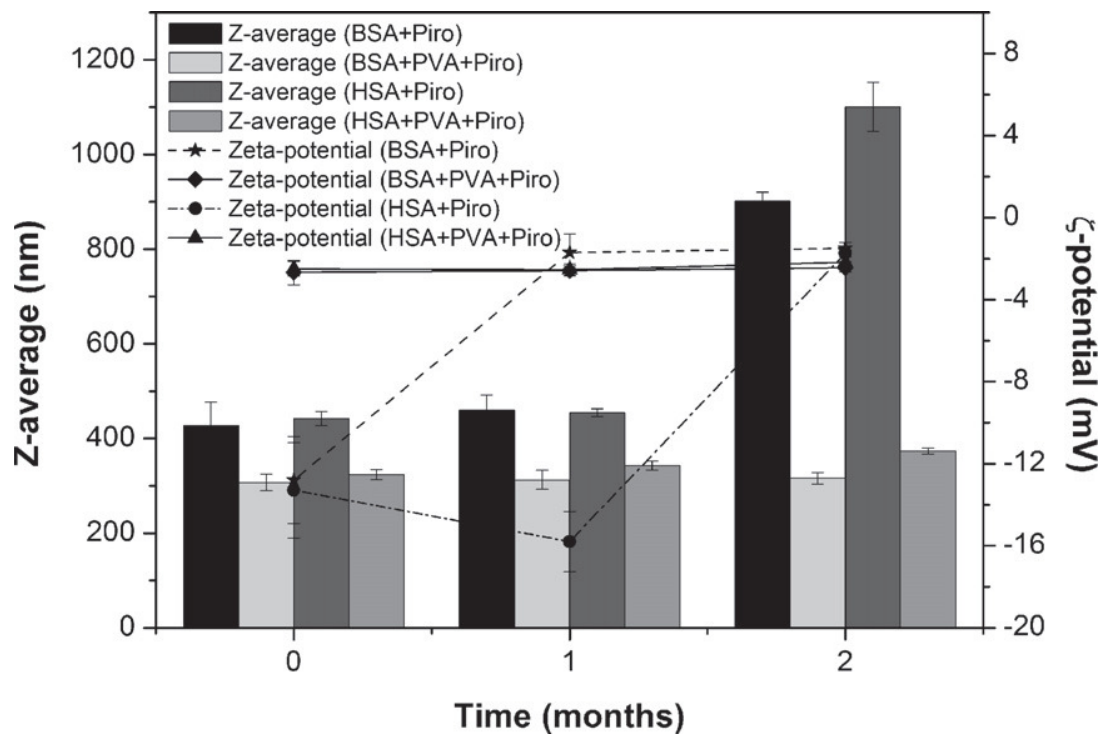

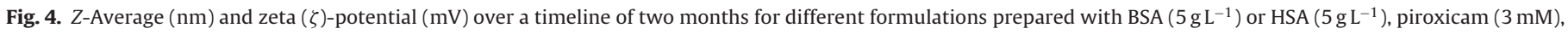
with or without PVA (8\%). Values reported are the mean \pm SD of at least three different batches of each microsphere formulation. 

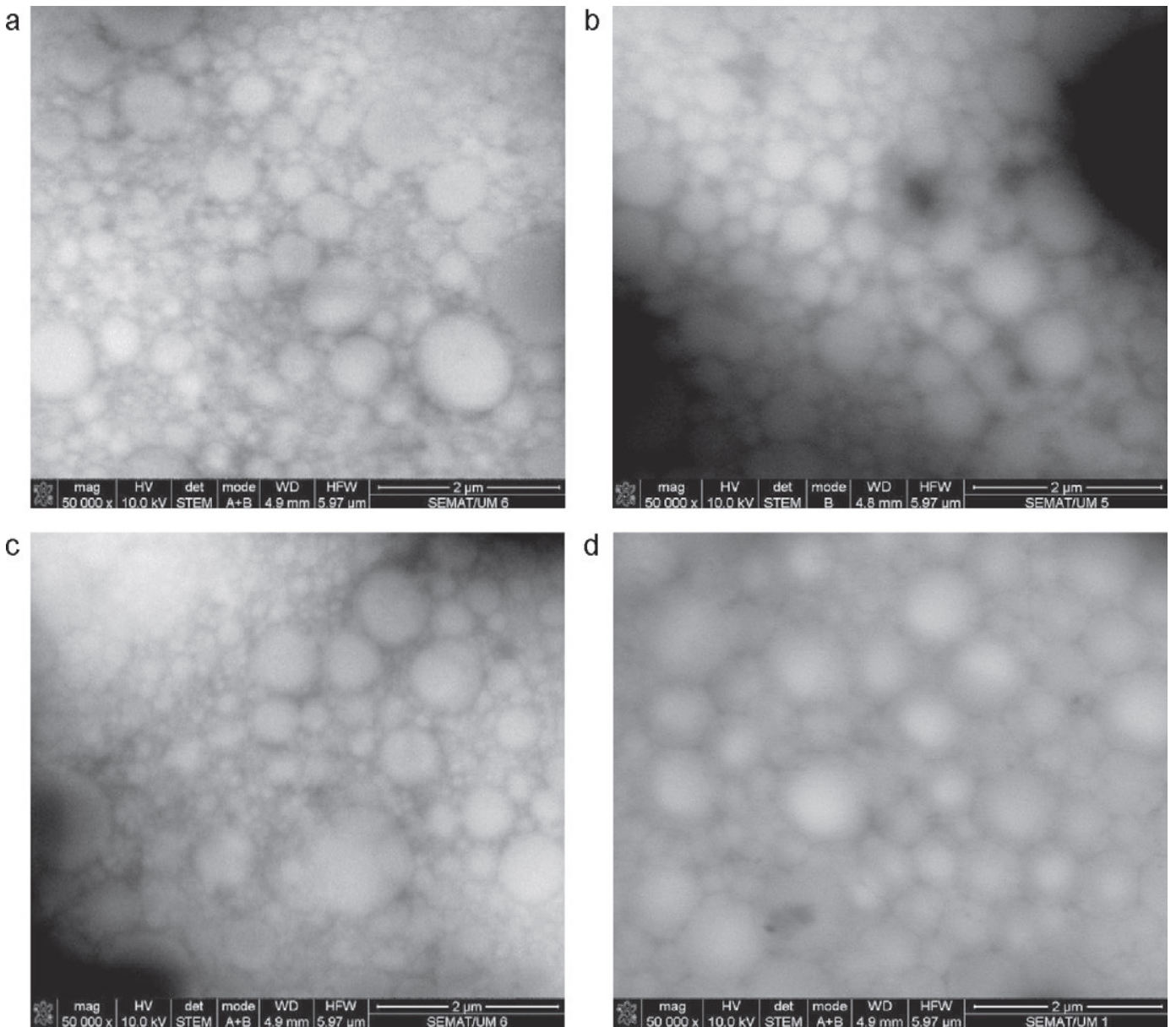

Fig. 5. STEM images ( $\times 50,000$ magnification) of protein microspheres: (a) BSA $\left(5 \mathrm{~g} \mathrm{~L}^{-1}\right)+$ piroxicam ( $\left.3 \mathrm{mM}\right)$; (b) BSA (5 g L $\left.{ }^{-1}\right)+\mathrm{PVA}^{(}(8 \%)+$ piroxicam ( $\left.3 \mathrm{mM}\right)$; (c) HSA $\left(5 \mathrm{~g} \mathrm{~L}^{-1}\right)+$ piroxicam $(3 \mathrm{mM}) ;(\mathrm{d}) \mathrm{HSA}\left(5 \mathrm{~g} \mathrm{~L}^{-1}\right)+\operatorname{PVA}(8 \%)+$ piroxicam $(3 \mathrm{mM})$.

$K_{\mathrm{p}}$ value [43]. Additionally, location studies in liposomes showed that piroxicam can be positioned with their hydrophilic part oriented toward the polar part of membrane and their hydrophobic segment in the upper part of the lipophilic tails [43]. Thus, the piroxicam, which is sparingly soluble in water, must be aligned between the albumin molecules, in the protein layer contributing also for the initial burst release. It was possible to verify that BSA particles display a faster release of piroxicam, compared with the release obtained with HSA microspheres. This result can be attributed to the fact that smaller particles have a larger surface area, therefore, most of the associated drug would be at or near the particle surface leading to a fast drug release. On the other hand, the observed higher sizes of HSA particles, can explain the longer sustained release compared to smaller particles. Different concentrations of protease $\left(0.073,0.50\right.$ and $\left.3.0 \mathrm{U} \mathrm{mL}^{-1}\right)$ were used for piroxicam release trigger on protein devices and the results of Fig. 6 evidence that the higher concentration lead to the most rapid diffuse out of drug.

A number of mathematical models have been proposed to describe the mechanisms of drug release from polymeric devices. The Higuchi [44], Korsmeyer [45] and Peppas [46,47] equations are the most widely used to date and are mainly based on the Fickian diffusion equation. In order to analyze the release mechanism of piroxicam from the proteinaceous microspheres and the effect of the protease concentrations over the release behavior, the obtained data was processed using the empirical expression proposed by Ritger-Peppas [46]. For a sphere, a Fickian diffusion of first-order is observed when $n$ has the limiting value of 0.43 ; if $n=0.85$ occurs Case II transport (polymer relaxation/degradation), leading to zero-order release. Finally, when $n$ lies between 0.43 and 0.85 , Anomalous transport is observed coupling Fickian diffusion and polymer degradation $[46,47]$.

In HSA microspheres (Table 3), the drug transport mechanism obtained for all experimental conditions was Non-Fickian diffusion or Anomalous (indication of the superposition of both extreme phenomena: drug diffusion and macromolecular chain relaxation/degradation). This result indicates that neither absolute Fickian diffusion (as result of pure drug diffusion) nor Case II-zero order (because of polymer chain relaxation/degradation) was the predominant mechanism in this case. Therefore, it can be concluded that the protease concentration does not significantly influences the drug transport mechanism, affecting solely the diffusion coefficient value. In Table 3 is possible to observe that BSA microspheres in the presence of higher protease concentrations present a higher $n$ value indicating the Case II-zero order nature, which means that the piroxicam release is mainly due to the macromolecular chain relaxation indicating the degradation of BSA microspheres. The proteins chemical nature reveal a significant influence in the drug transport mechanism (Anomalous and Case II-zero order nature), when higher enzyme concentrations are used, affecting the diffusion coefficient value. Moreover, correlation coefficient " $R^{2}$ " is above the permissible range, that is, 0.95, further supporting the validity of the results. Therefore, these proteinaceous microspheres evidence their potential, for example, in topical 

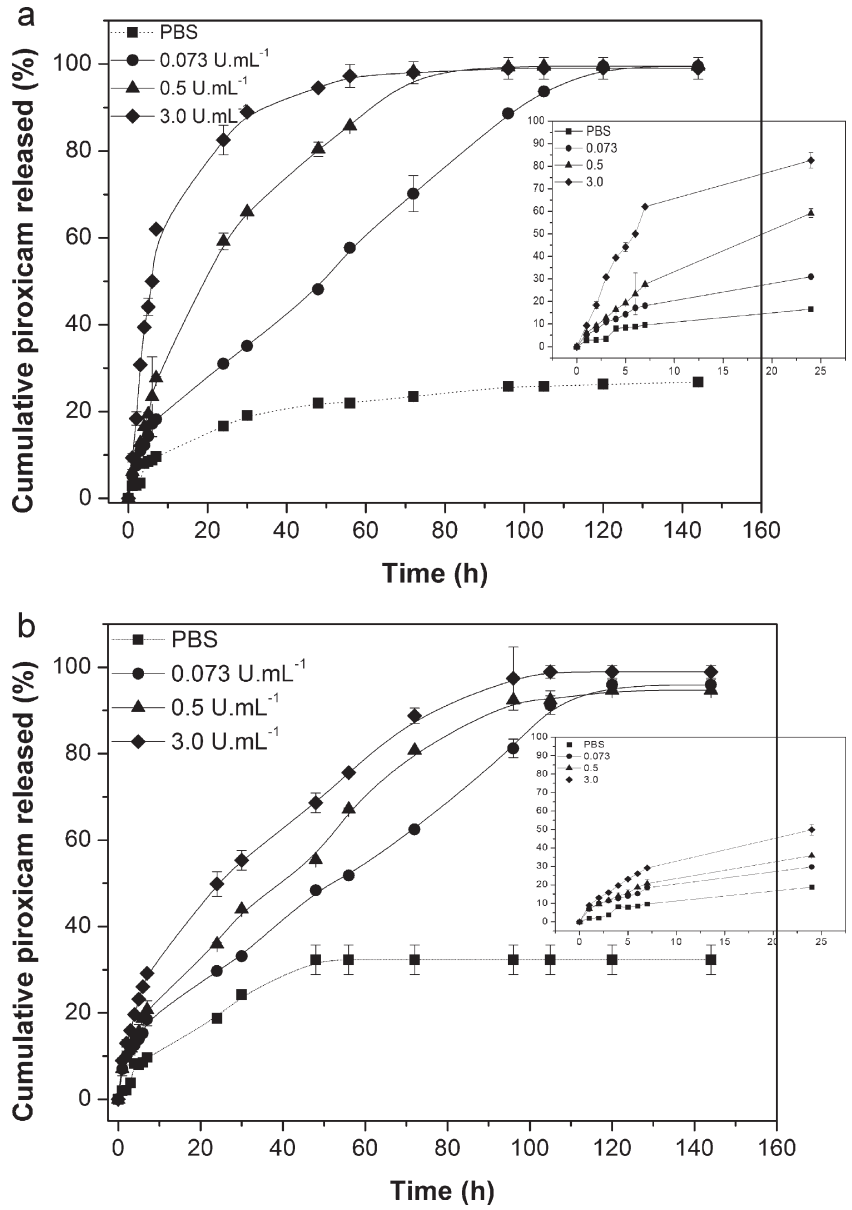

Fig. 6. In vitro release profile of piroxicam from BSA (a) and HSA (b) microspheres incubated with different concentrations of protease $\left(0.073,0.50\right.$ and $\left.3.0 \mathrm{U} \mathrm{mL}^{-1}\right)$ and with PBS buffer solution (control). Each point represents the mean \pm SD values from three different batches. The inset values show the release profile over the first $24 \mathrm{~h}$.

applications in the treatment of diseases where anti-inflammatory drugs are needed, such as in chronic wounds $[15,48]$. Additionally, the concentration of piroxicam that was released after $5 \mathrm{~min}$ (approximately $99 \mu \mathrm{M}$ or $33 \mu \mathrm{g} \mathrm{mL}^{-1}$ for PBS and in presence of $3 \mathrm{U}$ of protease) and $24 \mathrm{~h}$ (approximately $367 \mu \mathrm{M}$ or $120 \mu \mathrm{g} \mathrm{mL}^{-1}$ and $1780 \mu \mathrm{M}$ or $590 \mu \mathrm{g} \mathrm{mL}^{-1}$ for PBS and in presence of $3 \mathrm{U}$ of protease,
Table 3

Piroxicam release kinetic data obtained from fitting experimental release data to Ritger-Peppas Equation (4), where " $n$ " is the diffusion exponent and $R^{2}$ is the correlation coefficient.

\begin{tabular}{|c|c|c|c|c|}
\hline \multirow[t]{2}{*}{ Protease $\left(\mathrm{U} \mathrm{mL}^{-1}\right)$} & \multicolumn{2}{|l|}{ BSA } & \multicolumn{2}{|l|}{ HSA } \\
\hline & $n$ & $R^{2}$ & $n$ & $R^{2}$ \\
\hline 0.073 & 0.568 & 0.987 & 0.529 & 0.988 \\
\hline 0.50 & 0.699 & 0.994 & 0.526 & 0.996 \\
\hline 3.0 & 0.859 & 0.987 & 0.544 & 0.993 \\
\hline
\end{tabular}

respectively), can be enough to obtain a therapeutical effect. In fact, a study present in literature [50] shows that the concentration of piroxicam is in levels in the order of $0.3-4.6 \mathrm{mg} \mathrm{mL}^{-1}$ of synovial fluid, and in plasma the concentrations ranged between 0.5 and $8.3 \mathrm{mg} \mathrm{mL}^{-1}$.

\subsection{Cytotoxicity evaluation}

The biocompatibility of the piroxicam entrapped on proteinaceous devices was assessed in fibroblast cultures. A preliminary screening was made using murine embryonic fibroblasts cells (MEFs), as a measure of global cytotoxicity. In this study, a wide range of protein concentrations (from 75 to $5000 \mathrm{mg} \mathrm{L}^{-1}$ ) used for inhibition assays was evaluated and no toxicity was observed for the range concentrations of $75-300 \mathrm{mg} \mathrm{L}^{-1}$. The results obtained with MEFs were then confirmed with human skin fibroblasts, testing the range protein concentrations $\left(75-300 \mathrm{mg} \mathrm{L}^{-1}\right)$ that did not induced any damage to MEFs cells.

The effect of all tested conditions on BJ5ta was evaluated after 24,48 and $72 \mathrm{~h}$ of culture, in order to establish the effect of drug entrapped in proteinaceous microspheres (BSA and HSA) on exposed BJ5ta (Figs. 7 and 8). Results indicate that neither protein solutions (BSA and HSA), PVA solution (PVA Sol.) nor vegetable oil induce any toxicity for the investigated culture times $(P>0.05)$. However, it is clear that the effect of piroxicam, in a concentration of $0.5 \mathrm{mM}$, on human fibroblasts cells is statistically different when compared to control cells $\left({ }^{* * *} P<0.001\right)$ and with the other tested samples $(\cdots P<0.001)$. In Fig. 7 it is possible to verify that after $72 \mathrm{~h}$ of exposure to $300 \mathrm{mg} \mathrm{L}^{-1}$ of BSA microspheres containing piroxicam, the viability of BJ5ta decreased, suggesting moderate cytotoxicity effects when compared with cells control $\left({ }^{* *} P<0.01\right)$. Comparisons of different concentrations of BSA microspheres, containing piroxicam, with the other tested samples were also performed and statistically significant differences were

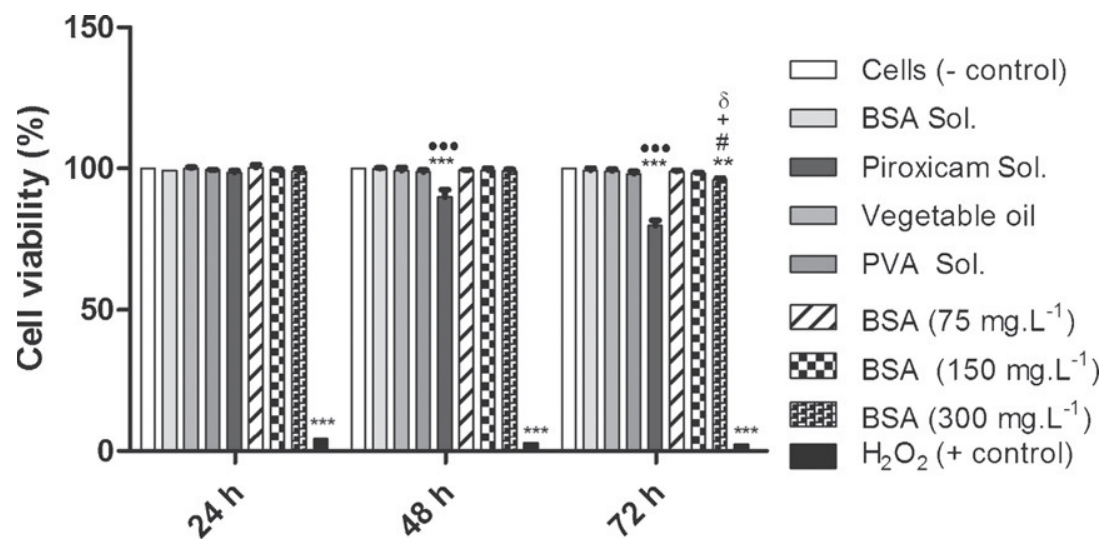

Fig. 7. BJ5ta cell viability at 24,48 and $72 \mathrm{~h}$ of culture with different solutions and different concentrations of BSA microspheres containing piroxicam ( 75,150 and $300 \mathrm{mg} \mathrm{L}^{-1}$ ). Values for tested samples are presented in relation to the control (cells cultured with culture medium scored $100 \%$ of viability). Statistically significant differences are indicated. *, significantly different from cells control; •, significantly different from other tested conditions; +, significantly different from BSA Sol.; \#, significantly different from Vegetable oil; $\delta$, significantly different from 75,150 and $300 \mathrm{mg} \mathrm{L}^{-1}$ of BSA microspheres. Results show the mean values of three replicates from two experiments carried out independently. 


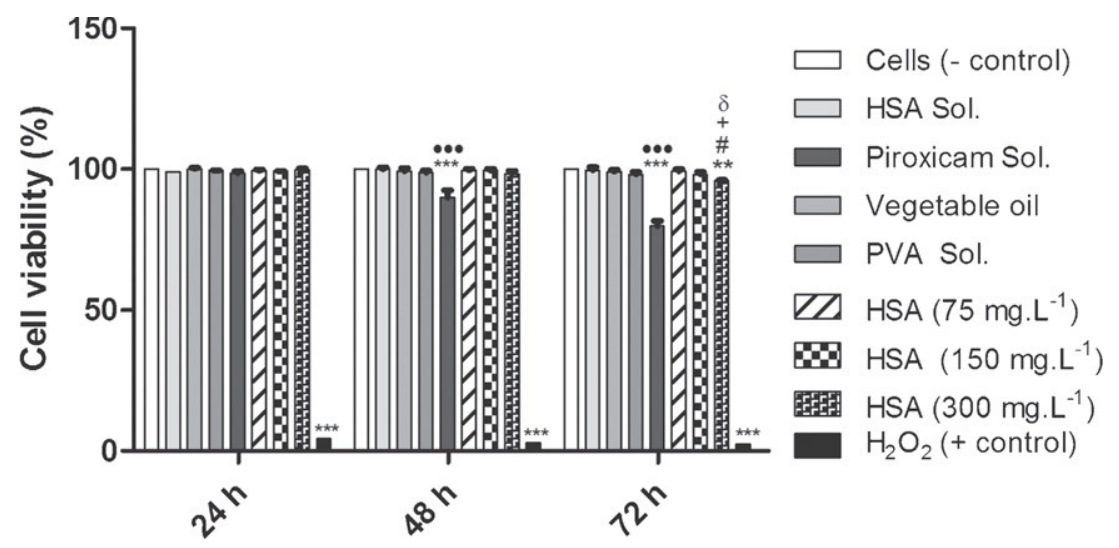

Fig. 8. BJ5ta cell viability at 24,48 and 72 h of culture with different solutions and different concentrations of HSA microspheres containing piroxicam ( 75,150 and 300 mg $\mathrm{L}^{-1}$ ). Values for tested samples are presented in relation to the control (cells cultured with culture medium scored $100 \%$ of viability). Statistically significant differences are indicated. *, Significantly different from cells control; •, significantly different from other tested conditions; +, significantly different from HSA Sol.; \#, significantly different from Vegetable oil; $\delta$, significantly different from 75,150 and $300 \mathrm{mg} \mathrm{L}^{-1}$ of HSA microspheres. Results show the mean values of three replicates from two experiments carried out independently.

obtained for the $300 \mathrm{mg} \mathrm{L}^{-1}$ concentration, after $72 \mathrm{~h}$ (+ symbol: BSA Sol. vs. $300 \mathrm{mg} \mathrm{L}^{-1} \mathrm{BSA}$; \# symbol: Vegetable oil vs $300 \mathrm{mg} \mathrm{L}^{-1}$ BSA; $\delta$ symbol: 150 and $75 \mathrm{mg} \mathrm{L}^{-1}$ BSA vs $300 \mathrm{mg} \mathrm{L}^{-1} \mathrm{BSA}, P<0.05$ ).

In Fig. 8, similar data is displayed for the viability of BJ5ta cells in contact with HSA microspheres, containing piroxicam, which further provides the evidence of cytotoxic effects after $72 \mathrm{~h}$, when $300 \mathrm{mg} \mathrm{L}^{-1}$ of HSA microspheres concentration is used (+ symbol: BSA Sol. vs $300 \mathrm{mg} \mathrm{L}^{-1}$ BSA; \# symbol: Vegetable oil vs $300 \mathrm{mg} \mathrm{L}^{-1}$ BSA; $\delta$ symbol: 150 and $75 \mathrm{mg} \mathrm{L}^{-1}$ BSA vs $300 \mathrm{mg} \mathrm{L}^{-1} \mathrm{BSA}, P<0.05$ and ${ }^{* *}$ symbol: Cells vs $300 \mathrm{mg} \mathrm{L}^{-1}$ BSA $P<0.01$ ). Statistically, it is possible to observe that the range concentrations of $75-150 \mathrm{mg} \mathrm{L}^{-1}$ of proteinaceous microspheres containing piroxicam, did not significantly affect the viability of human skin fibroblasts cells for a period of $72 \mathrm{~h}$, indicating that microspheres can be safely applied at these concentrations.

\section{Conclusion}

The present study demonstrates the ability of proteinaceous microspheres to entrap the anti-inflammatory drug piroxicam. BSA and HSA microspheres were produced with predictable and reproducible size by a sonochemical method. Furthermore, the incorporation of PVA into the prepared formulations resulted in improved characteristics, including smaller size, lower size distribution, higher stability and higher entrapment efficiency of piroxicam. The kinetics of piroxicam entrapped in BSA or HSA microspheres release mechanism was shown to be dependent on the protein and on protease concentration. The fast release is achieved when the drug is entrapped into BSA microspheres and in the presence of high protease concentration $\left(3.0 \mathrm{U} \mathrm{mL}^{-1}\right)$. However, if it is necessary a slower release the piroxicam should be incorporated in HSA microspheres. This fact, along with the cytocompatibility observed with human skin fibroblasts, indicates that these proteins may potentially be used as potential bioactive carriers for treatment of inflammatory diseases. In this sense, such drug carriers with a specific size range can be invaluable therapeutic tools for other pathologies where the NSAIDs can be useful, such as cancer, Alzheimer and artherosclerosis [1,51-53].

\section{Acknowledgements}

We would like to acknowledge the financial support of European project Lidwine (contract no. NMP2-CT-2006-026741), and to $\mathrm{POPH} / \mathrm{FSE}$ for co-financing and FCT for fellowship SFRH/BPD/38939/2007.

\section{References}

[1] M. Lúcio, H. Ferreira, J.L.F.C. Lima, S. Reis, Use of liposomes to evaluate the role of membrane interactions on antioxidant activity, Anal. Chim. Acta 597 (2007) $163-170$.

[2] J.C. Fröhlich, Prostaglandin endoperoxide synthetase isoenzymes: The clinical relevance of selective inhibition, Ann. Rheum. Dis. 54 (1995) 942-943.

[3] P. Brooks, Use and benefits of nonsteroidal anti-inflammatory drugs, Am. J. Med. 104 (1998) 9S-13S.

[4] W. Tomisato, K.-i. Tanaka, T. Katsu, H. Kakuta, K. Sasaki, S. Tsutsumi, T. Hoshino, M. Aburaya, D. Li, T. Tsuchiya, K. Suzuki, K. Yokomizo, T. Mizushima, Membrane permeabilization by non-steroidal anti-inflammatory drugs, Biochem. Biophys. Res. Commun. 323 (2004) 1032-1039.

[5] C. Goosen, J. du Plessis, D.G. Müller, L.F. Janse van Rensburg, Correlation between physicochemical characteristics, pharmacokinetic properties and transdermal absorption of NSAID's, Int. J. Pharm. 163 (1998) 203-209.

[6] P. Couvreur, L. Roblot-Treupel, M.F. Poupon, F. Brasseur, F. Puisieux, Nanoparticles as microcarriers for anticancer drugs, Adv. Drug Deliver. Rev. 5 (1990) 209-230.

[7] E. Allémann, R. Gurny, E. Doelker, Drug loaded nanoparticles: preparation methods and drug targeting issues, Eur. J. Pharm. Biopharm. 39 (1993) $173-191$.

[8] V.L. Truong-Le, S.M. Walsh, E. Schweibert, H.-Q. Mao, W.B. Guggino, J.T. August, K.W. Leong, Gene transfer by DNA-gelatin nanospheres, Arch. Biochem. Biophys. 361 (1999) 47-56.

[9] Y.-W. Won, Y.-H. Kim, Recombinant human gelatin nanoparticles as a protein drug carrier, J. Control. Release 127 (2008) 154-161.

[10] P. Couvreur, R. Gref, K. Andrieux, C. Malvy, Nanotechnologies for drug delivery: application to cancer and autoimmune diseases, Prog. Solid State Chem. 34 (2006) 231-235.

[11] O. Grinberg, M. Hayun, B. Sredni, A. Gedanken, Characterization, Activity of sonochemically-prepared BSA microspheres containing taxol - an anticancer drug, Ultrason. Sonochem. 14 (2007) 661-666.

[12] O. Grinberg, A. Gedanken, C.R. Patra, S. Patra, P. Mukherjee, D. Mukhopadhyay, Sonochemically prepared BSA microspheres containing gemcitabine, and their potential application in renal cancer therapeutics, Acta Biomater. 5(2009) 3031-3037.

[13] O. Grinberg, A. Gedanken, The development and characterization of starch microspheres prepared by a sonochemical method for the potential drug delivery of insulin, Macromol. Chem. Phys. 211 (2010) 924931.

[14] N. Skirtenko, T. Tzanov, A. Gedanken, S. Rahimipour, One-step preparation of multifunctional chitosan microspheres by a simple sonochemical method, Chem. Eur. J. 16 (2010) 562-567.

[15] R. Silva, H. Ferreira, A. Vasconcelos, A. Gomes, A. Cavaco-Paulo, Sonochemical proteinaceous devices for wound healing, Adv. Exp. Med. Biol. 733 (2011) 155-164.

[16] C. Weber, C. Coester, J. Kreuter, K. Langer, Desolvation process and surface characterisation of protein nanoparticles, Int. J. Pharm. 194 (2000) 91-102.

[17] B.G. Müller, H. Leuenberger, T. Kissel, Albumin nanospheres as carriers for passive drug targeting: an optimized manufacturing technique, Pharm. Res. 13 (1996) 32-37. 
[18] T. Peters Jr., J.T.E.C.B. Anfinsen, M.R. Frederic, Serum Albumin, Advances in Protein Chemistry, Academic Press, 1985, pp. 161-245

[19] M.L. Hans, A.M. Lowman, Biodegradable nanoparticles for drug delivery and targeting, Curr. Opin. Solid State Mater. Sci. 6 (2002) 319-327.

[20] Avivi, Y. Nitzan, R. Dror, A. Gedanken, An easy sonochemical route for the encapsulation of tetracycline in bovine serum albumin microspheres, J. Am. Chem. Soc. 125 (2003) 15712-15713.

[21] U. Shimanovich, I.D. Tkacz, D. Eliaz, A. Cavaco-Paulo, S. Michaeli, A. Gedanken, Encapsulation of RNA molecules in BSA microspheres and internalization into Trypanosoma brucei parasites and human U2OS cancer cells, Adv. Funct. Mater. 21 (2011) 3659-3666.

[22] O.H. Lowry, N.J. Rosebrough, A. Lewis Farr, R.J. Randall, Protein measurement with Folin-Phenol reagent, J. Biol. Chem. 193 (1951) 265-275.

[23] H. Bunjes, Characterization of solid lipid nanoparticles and microparticle, in: C. Nastruzzi (Ed.), Lipospheres in Drug Targets and Delivery Approaches, Methods and Applications, CRC Press LLC, Boca Raton, FL, 2005, pp. 41-66.

[24] K.S. Suslick, M.W. Grinstaff, Protein microencapsulation of nonaqueous liquids, J. Am. Chem. Soc. 780 (1990) 7-7809.

[25] R. Silva, H. Ferreira, R. Araujo, N. Azoia, A. Rollet, U. Angel, A. Gomes, G. Freddi, G. Güebitz, A. Gedanken, A. Cavaco-Paulo, Insights on the mechanism of protein microspheres formation, submitted for publication.

[26] Y.J. Wang, M.A.A. Hanson, Parenteral Drug. Parenteral Formulations of Proteins and Peptides: Stability and Stabilizers, Parenteral Drug Association, 1988.

[27] P. Ahlin, J. Kristl, A. Krist, F. Vrecer, Investigation of polymeric nanoparticles as carriers of enalaprilat for oral administration, Int. J. Pharm. 239 (2002) 113-120.

[28] T. Feczkó, J. Tóth, J. Gyenis, Comparison of the preparation of PLGA-BSA nanoand microparticles by PVA, poloxamer and PVP, Colloids Surf. A 319 (2008) $188-195$.

[29] M.N.V. Ravi Kumar, U. Bakowsky, C.M. Lehr, Preparation and characterization of cationic PLGA nanospheres as DNA carriers, Biomaterials 25 (2004) 1771-1777.

[30] J. Chen, E. Dickinson, Protein/surfactant interfacial interactions. Part 1. Flocculation of emulsions containing mixed protein + surfactant, Colloids Surf. A 100 (1995) 255-265.

[31] G. Chansiri, R.T. Lyons, M.V. Patel, S.L. Hem, Effect of surface charge on the stability of oil/water emulsions during steam sterilization, J. Pharm. Sci. 88 (1999) 454-458.

[32] S. Azarmi, Y. Huang, H. Chen, S. McQuarrie, D. Abrams, W. Roa, W. Finlay, G. Miller, R. Löbenberg, Optimization of a two-step desolvation method for preparing gelatin nanoparticles and cell uptake studies in 143B osteosarcoma cancer cells, J. Pharm. Pharmaceut. Sci. 9 (2006) 124-132.

[33] N. Mandzy, E. Grulke, T. Druffel, Breakage of $\mathrm{TiO}_{2}$ agglomerates in electrostatically stabilized aqueous dispersions, Powder Technol. 160 (2005) 121-126.

[34] J.M. Berg, A. Romoser, N. Banerjee, R. Zebda, C.M. Sayes, The relationship between $\mathrm{pH}$ and zeta-potential of $\sim 30 \mathrm{~nm}$ metal oxide nanoparticle suspensions relevant to in vitro toxicological evaluations, Nanotoxicology 3 (2009) 276-283.

[35] Y. Zhang, M. Yang, N. Portney, D. Cui, G. Budak, E. Ozbay, M. Ozkan, C. Ozkan, Zeta-potential: a surface electrical characteristic to probe the interaction of nanoparticles with normal and cancer human breast epithelial cells, Biomed. Microdevices 10 (2008) 321-328.
[36] B. Heurtault, P. Saulnier, B. Pech, J.-E. Proust, J.-P. Benoit, Physico-chemical stability of colloidal lipid particles, Biomaterials 24 (2003) 4283-4300.

[37] G.P. Yampolskaya, B.N.Tarasevich, A.A. Elenskii, Secondary structure of globular proteins in adsorption layers at the solution-air interface by the data of fourier transform IR spectroscopy, Colloid J. 67 (2005) 385-391.

[38] R. Silva, H. Ferreira, N. Azoia, A. Rollet, U. Shimanovich, G. Güebitz, A. Gedanken, A. Cavaco-Paulo, Mechanisms of the formation of proteinaceous microspheres - highlights from peptide size and sequences, submitted for publication.

[39] Y. Xu, Y. Du, Effect of molecular structure of chitosan on protein delivery properties of chitosan nanoparticles, Int. J. Pharm. 250 (2003) 215-226.

[40] I. Bala, S. Hariharan, M.N. Kumar, PLGA nanoparticles in drug delivery: the state of the art, Crit. Rev. Ther. Drug Carrier Syst. 21 (2004) 387-422.

[41] I. Bala, V. Bhardwaj, S. Hariharan, J. Sitterberg, U. Bakowsky, M.N.V.R. Kumar, Design of biodegradable nanoparticles: a novel approach to encapsulating poorly soluble phytochemical ellagic acid, Nanotechnology 16 (2005) 2819-2822.

[42] C. Dai, B. Wang, H. Zhao, B. Li, J. Wang, Preparation and characterization of liposomes-in-alginate (LIA) for protein delivery system, Colloids Surf. B 47 (2006) 205-210.

[43] M. Lúcio, H. Ferreira, J.L.F.C. Lima, S. Reis, Interactions between oxicams and membrane bilayers: an explanation for their different COX selectivity, Med. Chem. 2 (2006) 447-456.

[44] T. Higuchi, Rate of release of medicaments from ointment bases containing drugs in suspension, J. Pharm. Sci. 50 (1961) 874-875.

[45] R.W. Korsmeyer, R. Gurny, E. Doelker, P. Buri, N.A. Peppas, Mechanisms of solute release from porous hydrophilic polymers, Int. J. Pharm. 15 (1983) 25-35.

[46] P.L. Ritger, N.A. Peppas, A simple equation for description of solute release I. Fickian and non-fickian release from non-swellable devices in the form of slabs, spheres, cylinders or discs, J. Control. Release 5 (1987) 23-36.

[47] J. Siepmann, N.A. Peppas, Modeling of drug release from delivery systems based on hydroxypropyl methylcellulose (HPMC), Adv. Drug Deliver. Rev. 48 (2001) 139-157.

[48] I.T. Nizamutdinova, Y.M. Kim, J.I. Chung, S.C. Shin, Y.-K. Jeong, H.G. Seo, J.H. Lee, K.C. Chang, H.J. Kim, Anthocyanins from black soybean seed coats stimulate wound healing in fibroblasts and keratinocytes and prevent inflammation in endothelial cells, Food Chem. Toxicol. 47 (2009) 2806-2812.

[50] A.D. de Jager, H. Ellis, H.K.L. Hundt, K.J. Swart, A.F. Hundt, High-performance liquid chromatographic determination with amperometric detection of piroxicam in human plasma and tissues, J. Chromatogr. B: Biomed. Sci. Appl. 729 (1999) 183-189.

[51] G. Cao, R.L. Prior, Comparison of different analytical methods for assessing total antioxidant capacity of human serum, Clin. Chem. 44 (1998) 1309-1315.

[52] C. Behl, Alzheimer's disease and oxidative stress implications for novel therapeutic approaches, Prog. Neurobiol. 57 (1999) 301-323.

[53] L.L. De Zwart, J.H.N. Meerman, J.N.M. Commandeur, N.P.E. Vermeulen, Biomarkers of free radical damage: applications in experimental animals and in humans, Free Radical Biol. Med. 26 (1999) 202-226. 